\title{
Covid-19 Novel Corona Virus Control and preventions In China and Discussing Their National Success to Back to Normal Life, While World Is Combatting the Deadly Viral Pandemic
}

\author{
Dr Ahsan Siddiqui, M.D, M.S.P.H (UK)* \\ Quality Management \& Patient Safety Department, General Directorate of Health, Riyadh Saudi Arabia \\ *Corresponding author: Dr Ahsan Ali Siddiqui, Consultant Epidemiologist, Quality Management \& Patient Safety \\ Department, General Directorate of Health, Riyadh Saudi Arabia
}

\section{ARTICLE INFO}

Received: 嵊 May 05, 2020

Published: May 18, 2020

Citation: Ahsan Ali Siddiqui. Covid-19 Novel Corona Virus Control and preventions In China and Discussing Their National Success to Back to Normal Life, While World Is Combatting the Deadly Viral Pandemic. Biomed J Sci \& Tech Res 27(4)-2020. BJSTR. MS.ID.004544.

Keywords: Novel Coronavirus Pneumonia; Traditional Chinese Medicine; Qingfei Paidu Decoction; 2019-nCoVl; COVID-19; Chinese Herbal Medicine; Middle East Respiratory Syndrome; Severe Acute Respiratory Syndrome; Chinese Medicine

\section{Abstract}

Background and Objective: This Article is about the Prevention strategies adopted by Chinese government and health department to contain the spread of COVID-19 novel corona virus in their community. Also, author would discuss their success in control and stop the spread of COVID-19 novel corona virus as the world is trying to reduce the spread and deaths caused by this deadly corona virus Pandemic.

Methods: The author of this article has studied the various articles presenting the research of their hospitals and cases with patients' treatment and other preventions. All the cases mostly test positive with COVID-19 were closely monitored, studied and analyzed and written here in the article. A literature review or narrative review is a type of review article. A literature review is a scholarly paper that presents the current knowledge including substantive findings as well as theoretical and methodological contributions to a particular topic (Wikipedia Article, 2020). The author of the article has chosen integrative literature review as a methodology for this research article.

Result: The results of the selection and reviewing the Articles for this research analysis publication shows marked positive results. The results show the Traditional Chinese Medicine with western medicine or alone provide robust immunity to patients to fight against COVID-19. The measurement and statistical analysis by SPSS 19 diagrammatic presentation shows that majority of the patients get successful treatment by Traditional Chinese Medicine with western medicine or alone.

Conclusion: The main theme of this Article is to discuss the Preventions, training and control strategies against COVID-19 novel corona virus in China in initial first two months of Pandemic. When Chinese health authorities in Wuhan china found Novel coronavirus pneumonia (NCP) in patients in health facilities they first treated them with (TCM) Traditional Chinese medicines with western medicines. In most of the cases they have found (TCM) Traditional Chinese medicines more effective than other medicines. The current positive statistics of Pandemics about China shows the complete control on COVID-19 with no spread and no Fatalities in May 2019. While the Pandemic of COVID-19 is on the rise all around the world specially in the USA, Europe and the UK.

\section{Introduction}

Through literature review and group discussion Special Expert Group for control of the Epidemic of Novel Coronavirus Pneumonia of the Chinese Preventive Medicine Association formulated an update on the epidemiological characteristics of novel coronavirus pneumonia (NCP) [1]. The initial source of the 2019 novel coronavirus (2019-nCoV) was the Huanan seafood market in Wuhan Hubei province China with pangolins as a potential animal host. Currently the main source of infection is NCP patients and 
asymptomatic carriers may also be infectious. The virus is believed transmitted mostly via droplets or contact. People are all generally susceptible to the virus. The average incubation period was 5.2 days and the basic reproductive number R (0) was 2.2 at the onset of the outbreak. Most NCP patients were clinically mild cases. The case fatality rate was $2.38 \%$, and elderly men with underlying diseases were at a higher risk of death. Strategies for prevention and control of NCP include improving epidemic surveillance quarantining the source of infection, speeding up the diagnosis of suspected cases, optimizing the management of close contacts, tightening prevention and control of cluster outbreaks and hospital infection, preventing possible rebound of the epidemic after people return to work from the Chinese Spring Festival holiday, and strengthening community prevention and control [1].

As of January, 22 2020, a total of 571 cases of the 2019new coronavirus (2019-nCoV) have been reported in 25 provinces (districts and cities) in China [2]. At present there is no vaccine or antiviral treatment for human and animal coronavirus so that identifying the drug treatment options as soon as possible is critical for the response to the 2019-nCoV outbreak. Three general methods which include existing broad-spectrum antiviral drugs using standard assays screening of a chemical library containing many existing compounds or databases and the redevelopment of new specific drugs based on the genome and biophysical understanding of individual coronaviruses are used to discover the potential antiviral treatment of human pathogen coronavirus. Lopinavir /Ritonavir, Nucleoside analogues, Neuraminidase inhibitors, Remdesivir, peptide (EK1), abidol, RNA synthesis inhibitors (such as TDF, 3TC), anti-inflammatory drugs (such as hormones and other molecules), Chinese traditional medicine, such ShuFengJieDu Capsules and Lianhuaqingwen Capsule, could be the drug treatment options for 2019-nCoV. However, the efficacy and safety of these drugs for 2019- nCoV still need to be further confirmed by clinical experiments [2].

The outbreak caused by 2019 novel coronavirus(2019-nCoV) is still spreading posing a great threat to the safety and health of general population. However there have not been any effective drugs for treatment with symptomatic treatment and prevention prevailing. The treatment plans of severe acute respiratory syndrome (SARS) and Middle East respiratory syndrome (MERS) are often used for reference in clinic. The advantages of traditional Chinese medicine(TCM) in treating SARS and MERS are that it can intervene and block the progression of disease in early stage significantly reduce symptoms, shorten the treatment duration of patients, reduce complications and side effects caused by hormone therapy [3]. The coronavirus disease 2019(COVID-19) belongs to the category of TCM epidemic diseases. Chinese patent medicines and prescriptions in medical observation and clinical treatment were recommended in the \& quot; pneumonia diagnosis and treatment plan for new coronavirus infection \& quot;(trial version fifth) of the National Health Commission of the People's Republic of China. Qingfei Paidu Decotion was recommended for the treatment of COVID-19 by the National Health Commission of the People's Republic of China and National Administration of Traditional Chinese Medicine. In this paper we reviewed traditional Chinese medicines and its active ingredients in the hope of bringing novel inspirations to the drug screening and clinical treatment for COVID-19 [3].

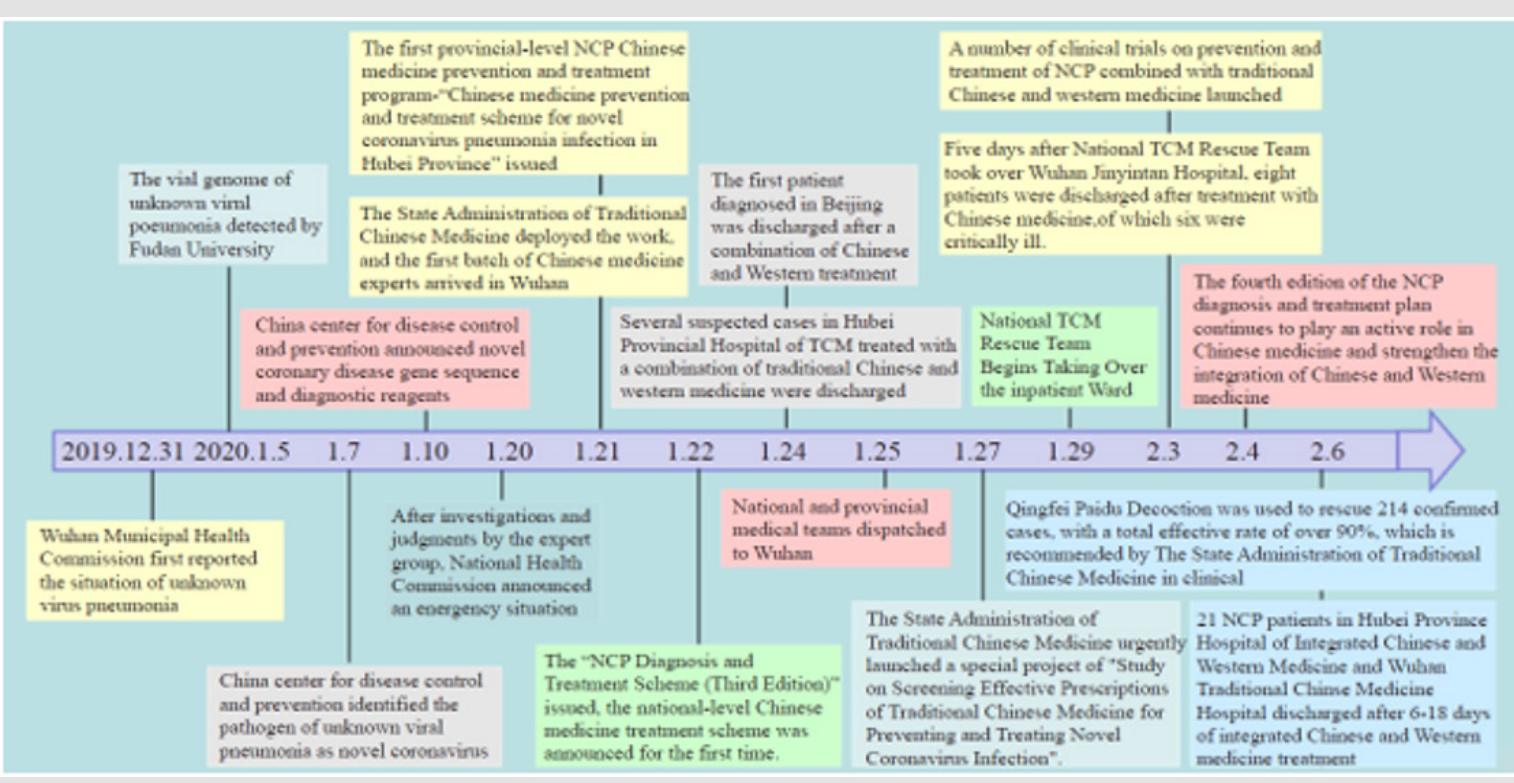

Figure 1: The actions and treatment of TCM on NCP in China (Hong-Zhi DU et al., 2020).

The novel coronavirus pneumonia broke out in 2019 and spread rapidly. In 30 different countries, there are over seventythousand patients have been diagnosed in total [2]. Therefore, it is urgent to develop the effective program to prevent and treat for the novel coronavirus pneumonia. In view of Traditional Chinese Medicine has accumulated a solid theoretical foundation of plague in ancientand recent decades. Meanwhile, Traditional Chinese Medicine can provide the more effective and personalized treatment 
via adjustingthe specific medicine for each patient based on the different syndromes. In addition TCM often has different effect on the distinctstages of diseases, contributing to the prevention, treatment and rehabilitation. Nowadays, TCM has exhibited decent effect in thein the fight against NCP. Therefore, it is convinced that Traditional Chinese Medicine is an effective treatment for 2019 novelcoronavirus pneumonia [2] (Figures 1 \& 2).

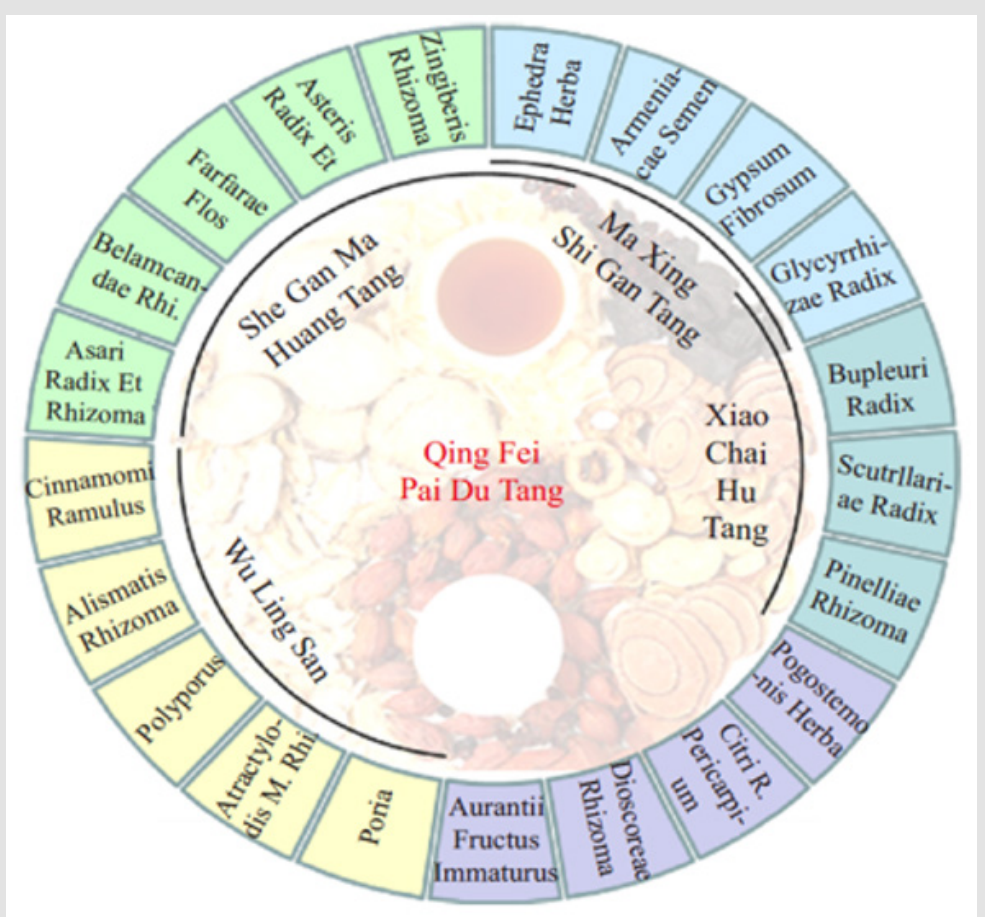

Figure 2: The composition of Prescription Qing Fei Pai Du Tag.

Incubation period of COVID19 is about 2-14 days but infected persons can transmit the virus via close contact and respiratory droplets perhaps even before they become symptomatic [4]. Physiological changes in the immune and respiratory system may make pregnant women more susceptible to COVID-19 infection during the epidemic. No effective vaccine is available at present. Therefore, it is advisable that pregnant women refrain from unnecessary travel, avoid crowds, public transport, contact with sick people, and more importantly, practice and maintain good personal and social hygiene. Pregnant women with symptoms of fever, cough, fatigue, myalgia, sore throat or shortness of breath should seek timely medical consultation and help. Women with a travel history to endemic areas and those with a clinical suspicion of infection should be isolated and investigated. Some pregnant women may develop severe anxiety and depression requiring professional psychological support to prevent adverse outcomes [4].

The clinical treatment and outcomes of the 55 patients were shown in Table. As of February, 15 2020, 26 patients (47.3\%) were still hospitalized whereas a total of 29 patients (52.7\%) had been discharged (Sun L, Shen L, Fan J et al, 2020). Significantly more severe cases received mechanical ventilation (non-invasive: $33.3 \%$ vs. $0 \%, p=0.001$; invasive: $20.0 \%$ vs.0\%, $\mathrm{p}=0.017$ ) as compared with non-severe cases. Only 1 patient $(12.5 \%)$ of those who received mechanical ventilation had been discharged while the other 7 patients $(87.5 \%)$ were still hospitalized at this point. 48 (87.3\%) patients received antiviral therapy, 51 (92.7\%) patients were given interferon alpha inhalation. $43(78.2 \%)$ cases received lopinavir/ritonavir treatment and 36 (65.5\%) patients were given lopinavir/ritonavir plus interferon alpha inhalation treatment. 29 (52.7\%) were given empirical antibiotic treatment 25 (45.5\%) were adopted systematic corticosteroid and 22 (40.0\%) received gamma globulin treatment. Moreover, blood purification therapy was adopted in $3(20.0 \%)$ severe cases but none in non-severe cases $(\mathrm{p}=0.004)$. Chinese medicine was given to $6(10.9 \%)$ cases and $1(1.8 \%)$ patient received convalescent plasma therapy [5]. On the basis of antiviral drugs, antibiotics treatment, appropriate dosage of corticosteroid and gamma globulin therapy significantly improve patients' outcomes $(\mathrm{p}<0.05)$.

For the request from frontline clinicians and public health professionals of 2019-nCoV infected pneumonia management an evidence-based guideline urgently needs to be developed [6]. Therefore, we drafted this guideline according to the rapid advice guidelines methodology and general rules of WHO guideline development we also added the firsthand management data of Zhongnan Hospital of Wuhan University. This guideline includes the guideline methodology, epidemiological characteristics, disease screening and population prevention, diagnosis, treatment and control (including traditional Chinese Medicine), nosocomial infection prevention and control, and disease nursing of the 2019- 
nCoV. Moreover, we also provide a whole process of a successful treatment case of the severe 2019-nCoV infected pneumonia and experience and lessons of hospital rescue for 2019-nCoV infections. This rapid advice guideline is suitable for the first frontline doctors and nurses, managers of hospitals and healthcare sections, community residents, public health persons, relevant researchers, and all person who are interested in the 2019-nCoV [6].

\section{Methods}

The author of this article has studied the various articles presenting the research of their hospitals and cases with patients treatment and other preventions. All the cases mostly test positive with COVID-19 were closely monitored, studied and analyzed and mentioned here in the article. A literature review or narrative review is a type of review article. A literature review is a scholarly paper that presents the current knowledge including substantive findings as well as theoretical and methodological contributions to a particular topic [7]. The author of the article has chosen integrative literature review as a methodology for this research article.

The main types of literature reviews are evaluative, exploratory, and instrumental. A fourth type, the systematic reviewis often classified separately but is essentially a literature review focused on a research question, trying to identify, appraise, select and synthesize all high-quality research evidence and arguments relevant to that question [7]. A meta-analysis is typically a systematic review using statistical methods to effectively combine the data used on all selected studies to produce a more reliable result. Torraco (2016) describes an integrative literature review the purpose of an integrative literature review is to generate new knowledge on a topic through the process of review, critique, and then synthesis of the literature under investigation [7].

Chinese medical sciences theory and clinical application of TCM were analyzed. A total of 54 NCP patients were observed in a hospital from Wuhan whose clinical characteristics and utilization of Chinese Medicines (CMs) were described [8]. Paired t test was used to measure the change of patients hemogram during hospitalization period indicating the effect of CMs. Multiple linear regression analysis was applied to explore the factors affecting the length of hospital stay. Network pharmacology analysis was applied to figure out the performance of NHC recommended formulas of five disease stages at levels of compounds targets and pathways. Clinical results of our study showed that 54 NCP patients enhanced immune ability against COVID-19 by detecting blood samples after TCM treatment [8] (Table 1 \& Figures 3-10).

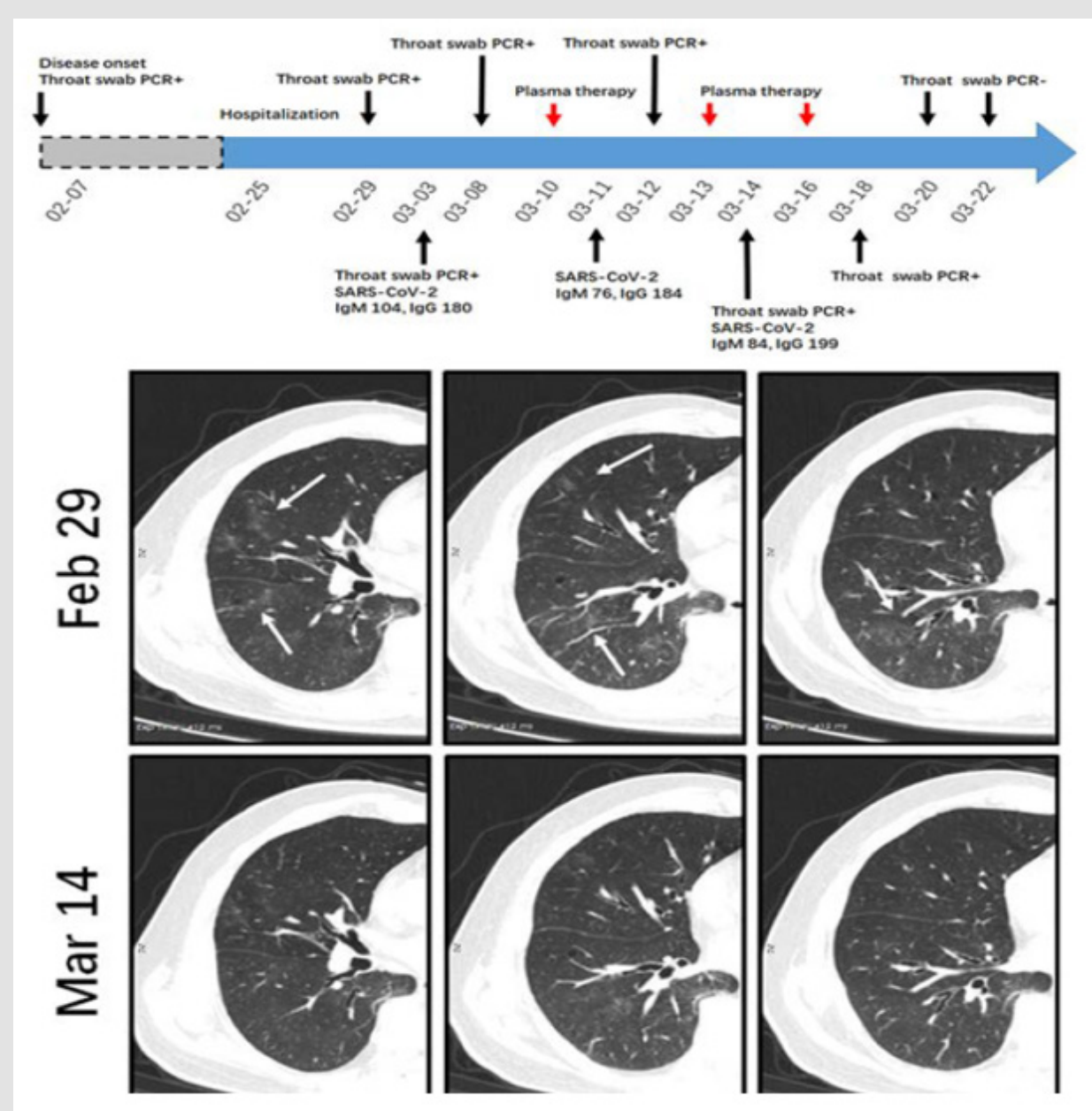

Figure 3: CT Scan 2 attached for Patient 2: (Wiley Library Journal of Medical Virology, 2020). 


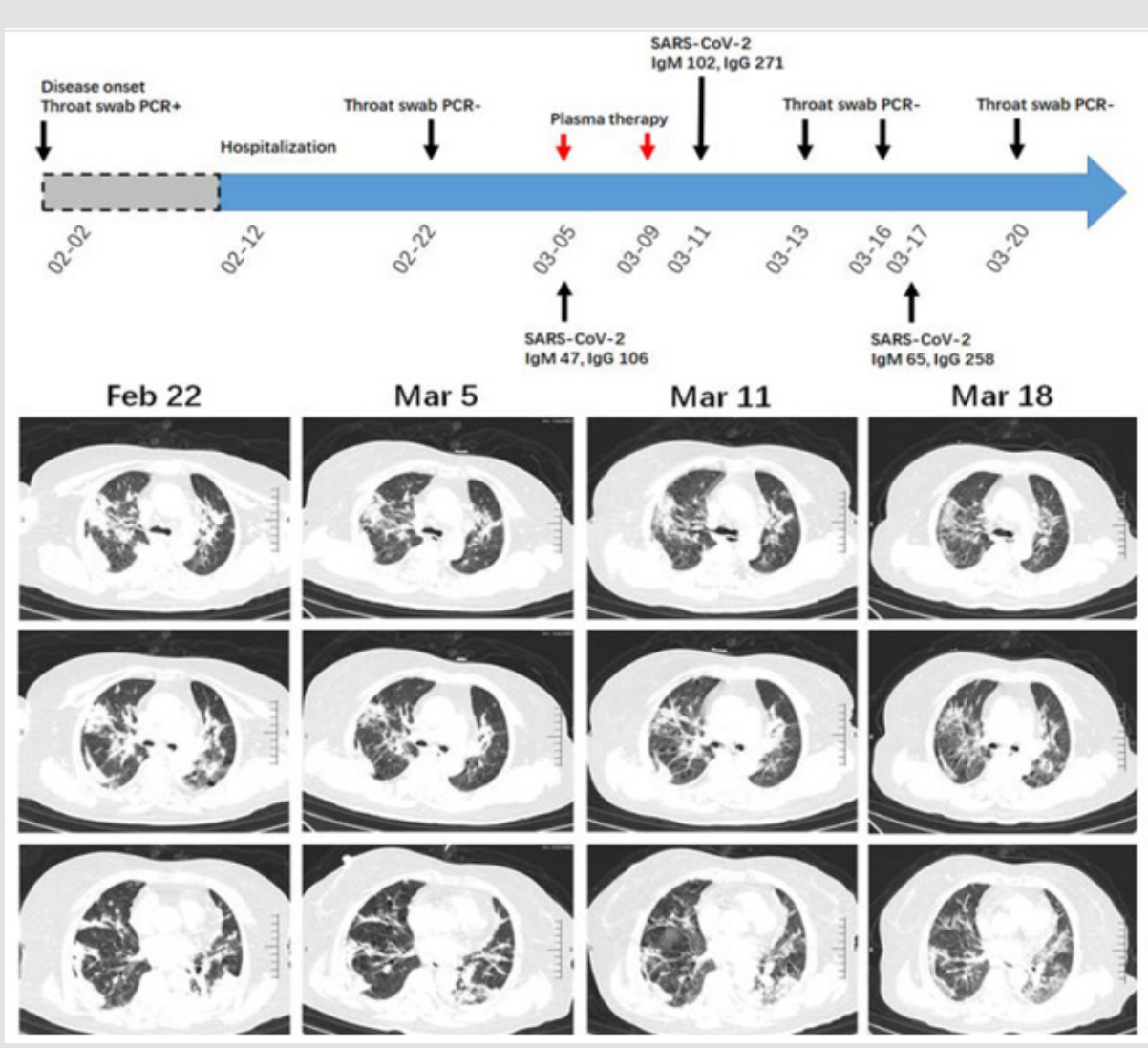

Figure 4: CT Scan 3 attached for Patient 3: (Wiley Library Journal of Medical Virology, 2020).

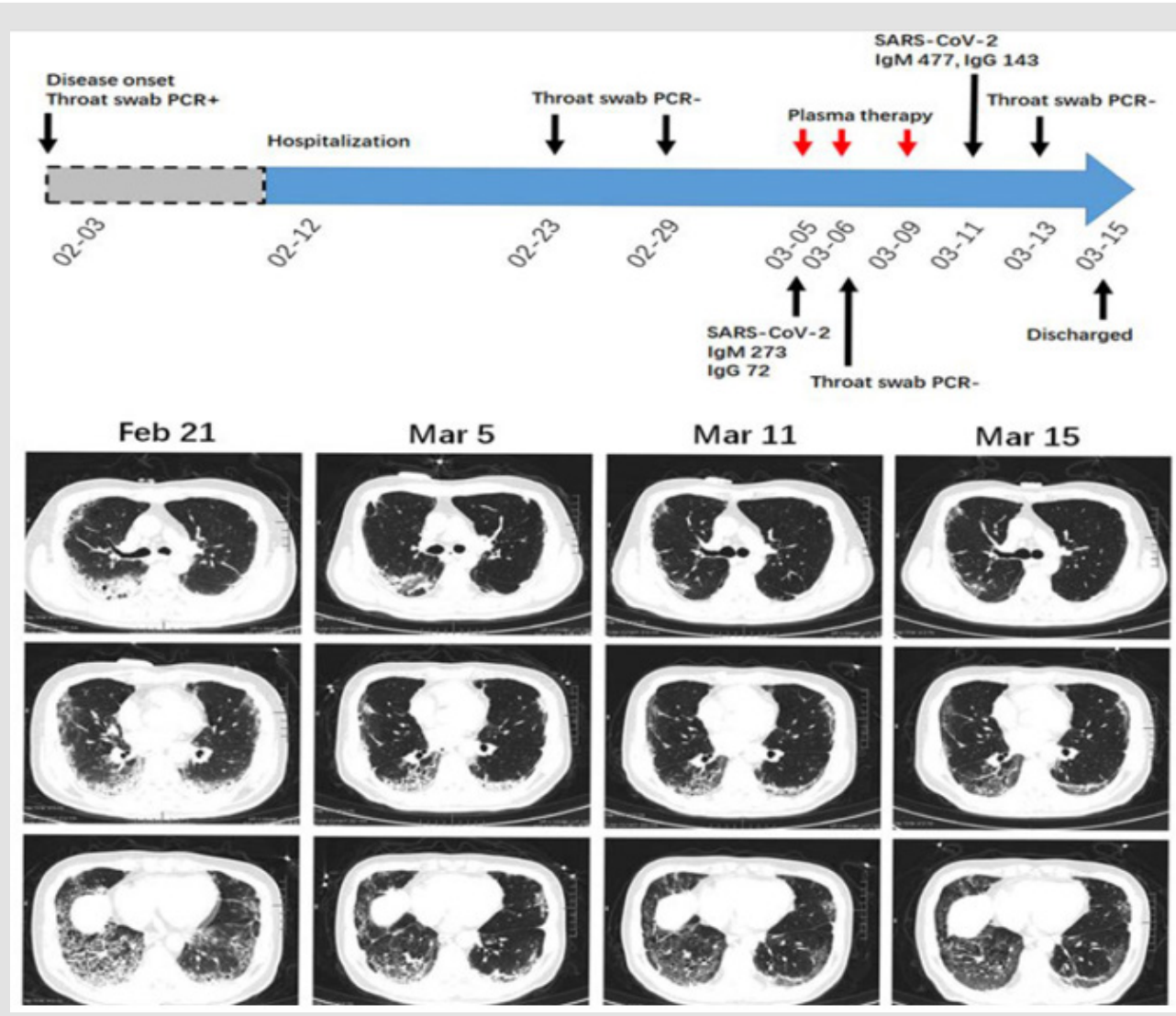

Figure 5: CT Scan 4 attached for Patient 4: (Wiley Library Journal of Medical Virology, 2020). 


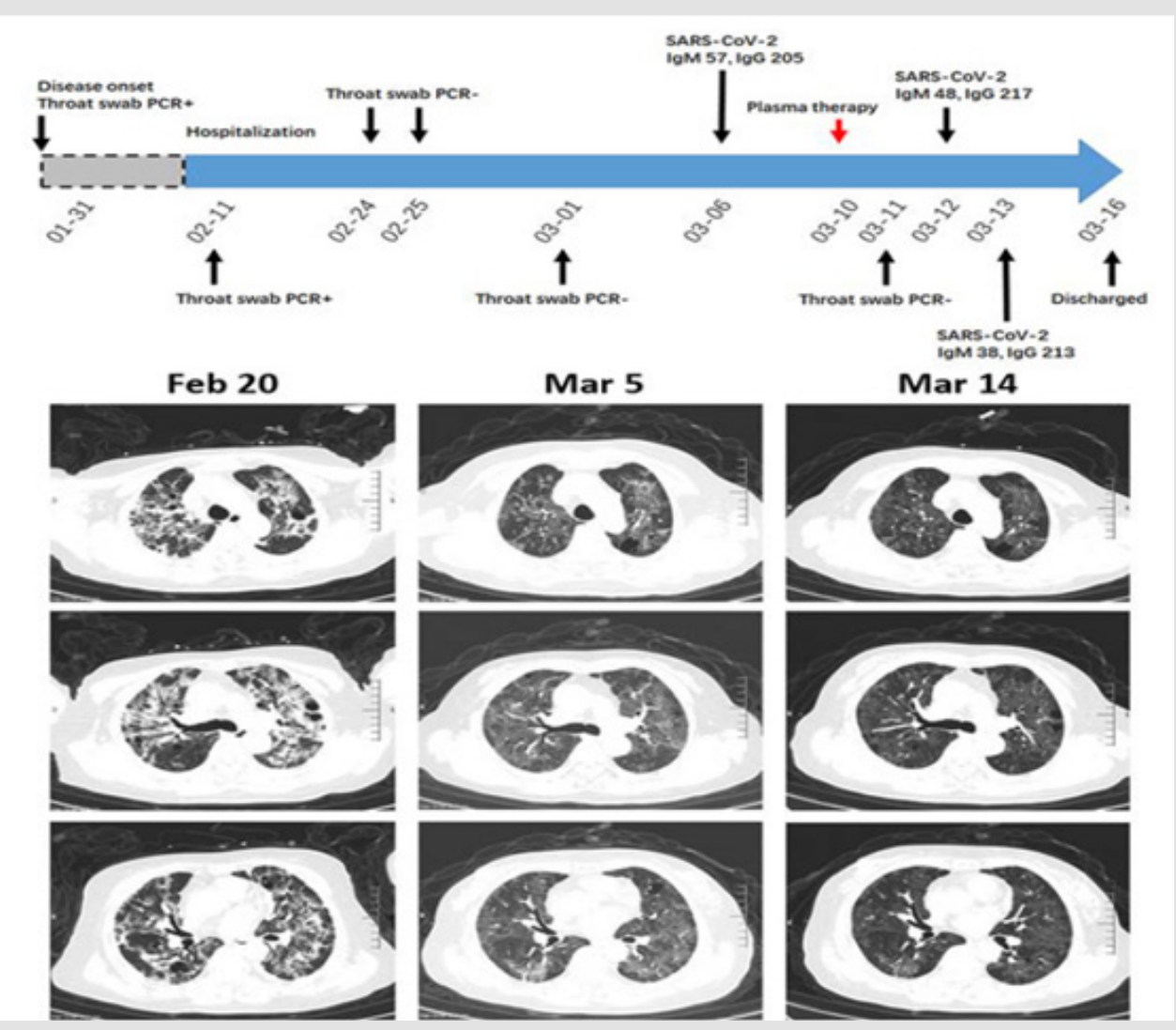

Figure 6.

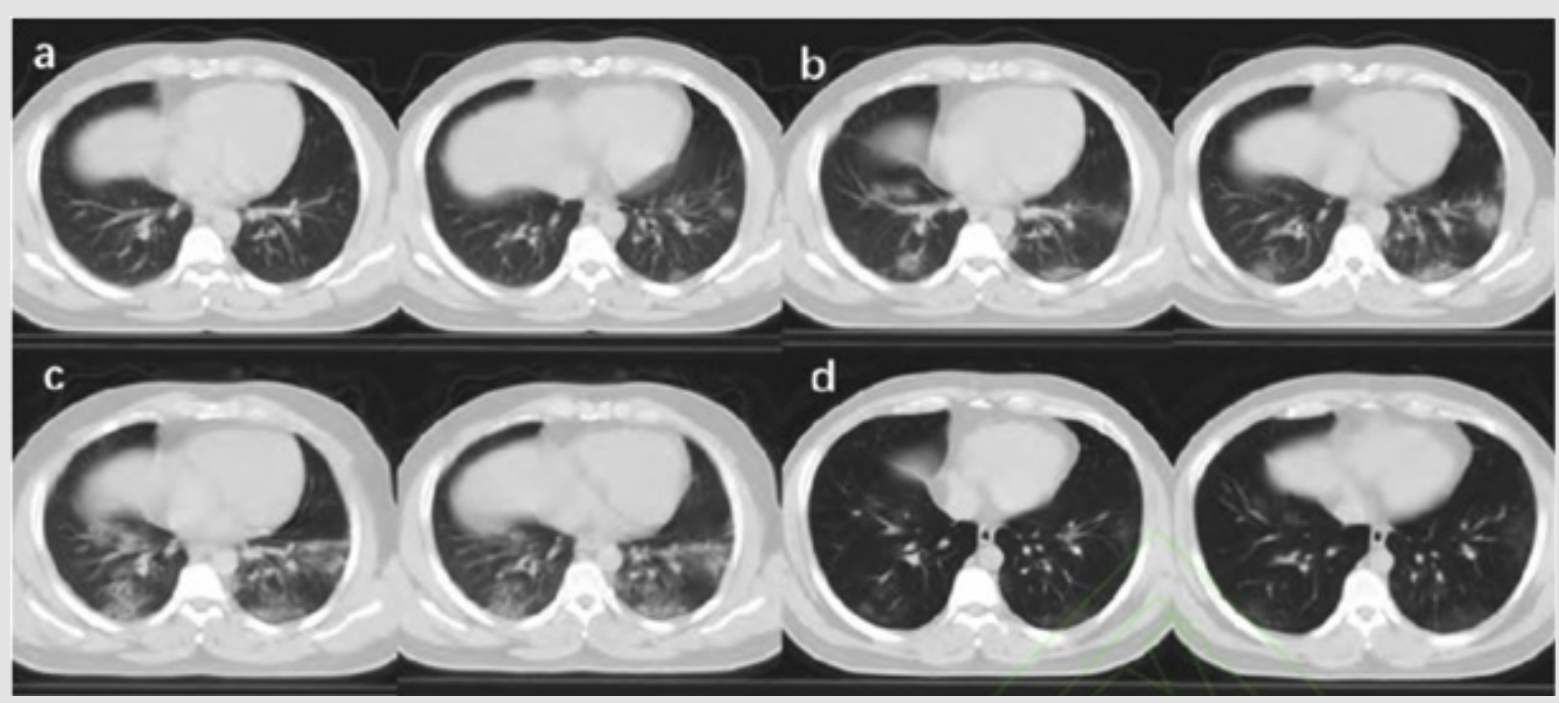

Figure 7: Measure and statistical Analysis:(IBM (2006) IBM SPSS Software).

Note: (ScienceDirect ELSEVIER, 2020): Figure below Comparison of chest CT results of patients on business trip in Wuhan China(a), chest CT on January 24; (b), chest CT on January 28; (c), chest CT on January 30; (d), chest CT on February 4 . Chest $\mathrm{CT}$ showed that the fusion of two lung ground glass shadows was enlarged and the density was increased, which was more advanced than that of admission (Fig. 1a-1c). After 6 days of treatment, chest CT was better than before, tracheobronchial shadow was normal, and inflammation was obviously absorbed (Fig. 1d)(ScienceDirect ELSEVIER, 2020). 


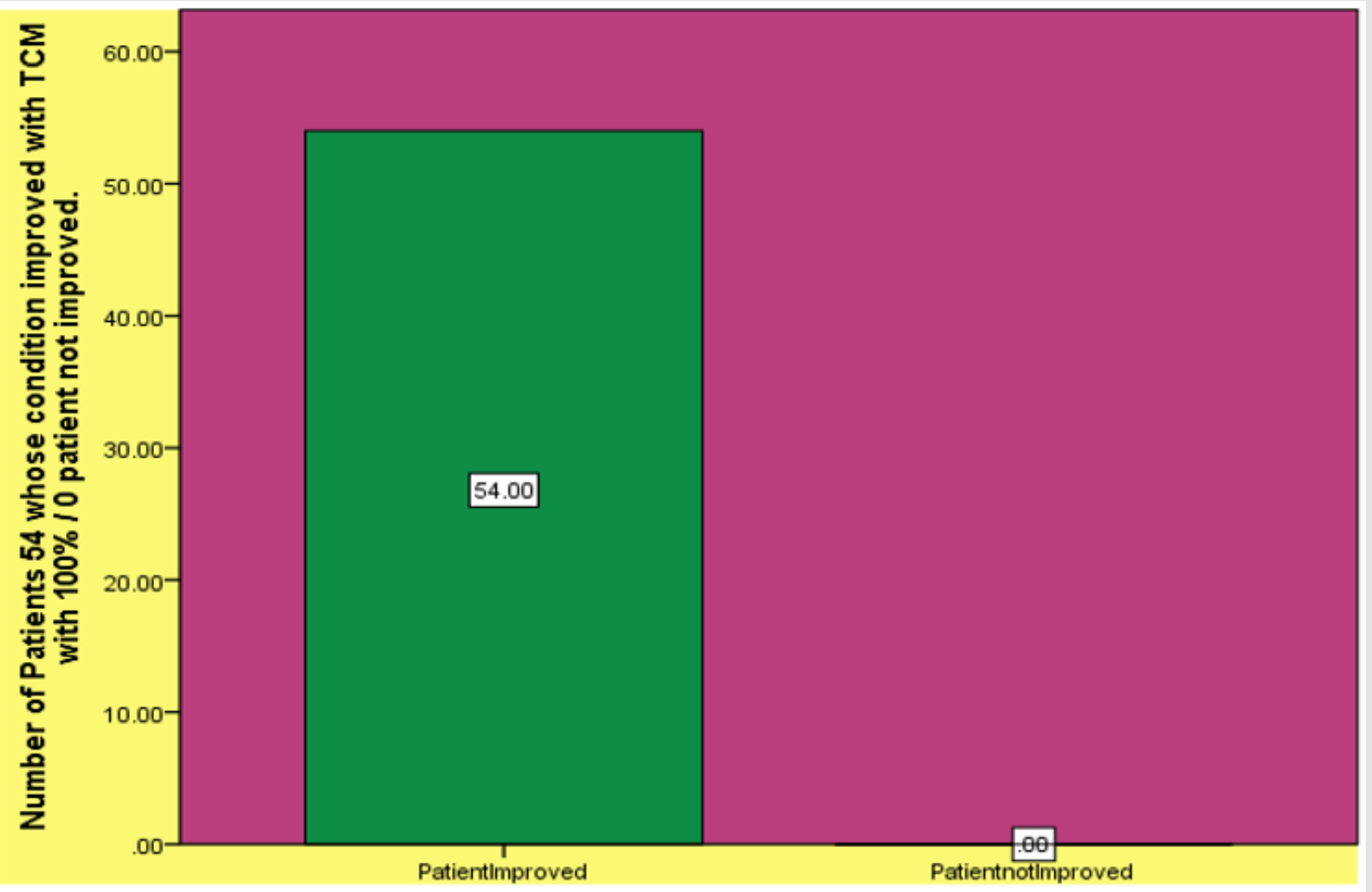

Figure 8: Luo E, Zhang D, Luo H, et al. (2020) the study of 54 patients shows 100 percent improvement in immune system after taking traditional Chinese medicines.

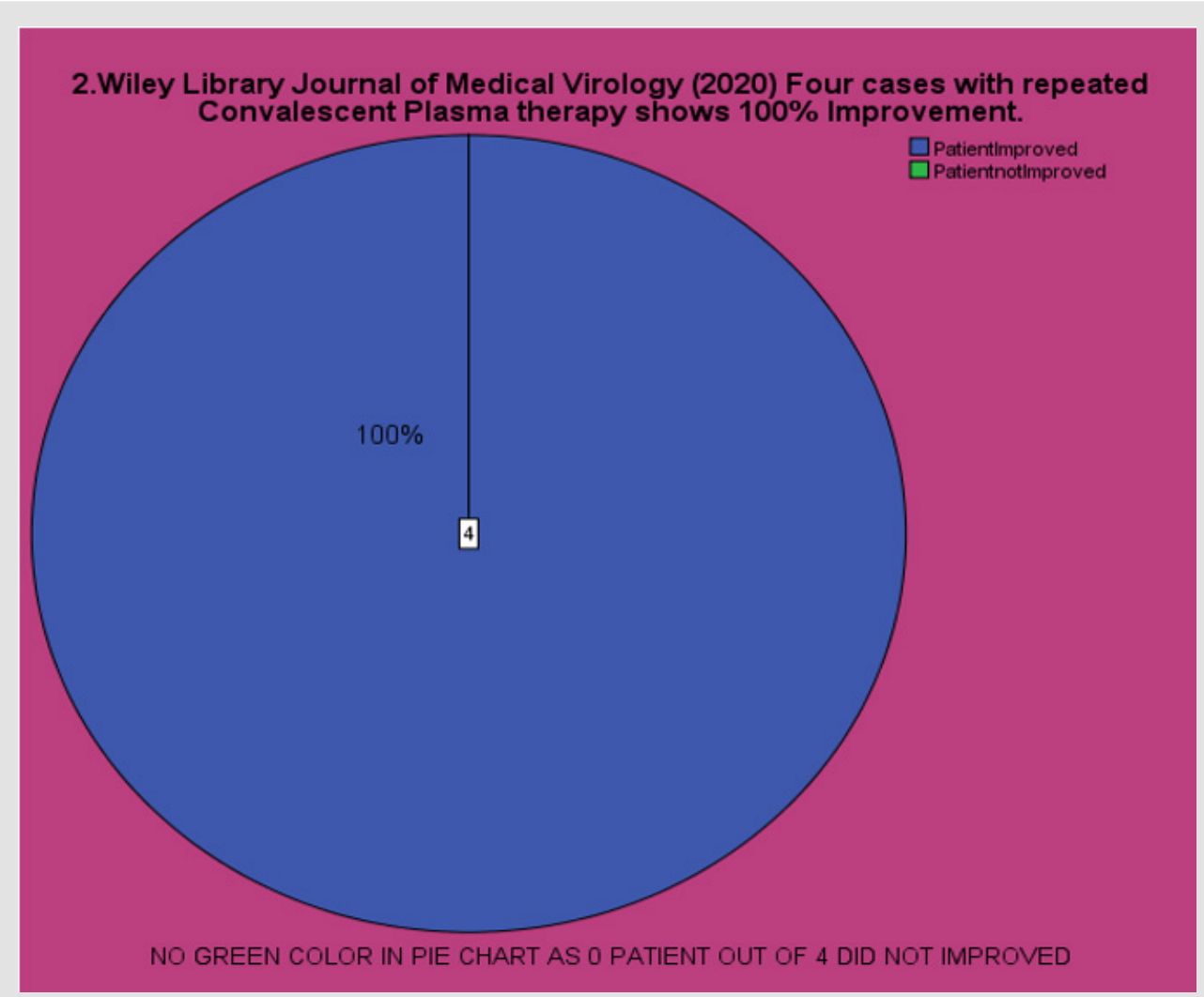

Figure 9: Wiley Library Journal of Medical Virology (2020) Four cases with repeated Convalescent Plasma therapy shows 100\% Improvement. 


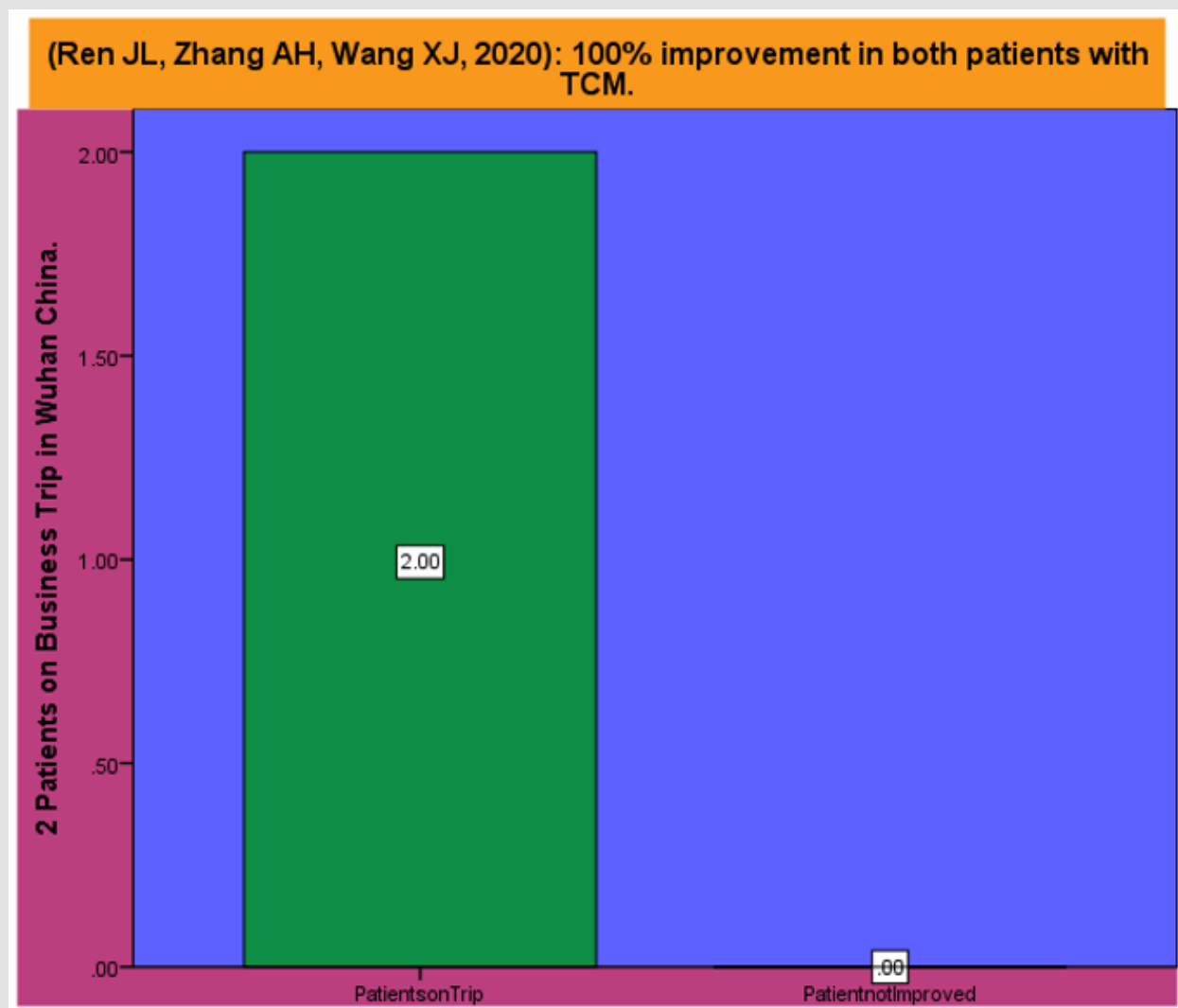

Figure 10: (Ren JL, Zhang AH, Wang XJ, 2020).

Table 1: CT Scan 1 attached for Patient 1: (Wiley Library Journal of Medical Virology, 2020).

Wiley Library Journal of Medical Virology (2020) Treatment with convalescent plasma for COVID-19 patients in Wuhan, China. Available at: https:// onlinelibrary.wiley.com/doi/abs/10.1002/jmv.25882 (Accessed on 02/05/2020). We would discuss 4 COVID-19 Medical Cases here treated in Hospital in Wuhan China.

\begin{tabular}{|c|c|}
\hline Case1 & $\begin{array}{l}\text { A } 69 \text { years old COVID-19 patient with persistent SARS-CoV-2 detection admitted to hospital in Wuhan china } \\
\text { and has received repeated transfusion of convalescent plasma and Levofloxacin. (Chest CT Scan } 1 \text { attached for } \\
\text { patient 1) Chest CT examination on February } 29^{\text {th }} \text { indicated patchy areas of GGOs in the right lung. Chest CT on } \\
\text { March } 14^{\text {th }} \text { indicated that the GGOs were resolved. }\end{array}$ \\
\hline Case 2 & $\begin{array}{l}\text { A } 75 \text {-year-old women COVID-19 patient with consolidation admitted in the Wuhan hospital. Her Chest CT scan } \\
\text { on February } 22^{\text {nd }} \text { showed multiple subpleural consolidation in bilateral lungs. After continuous deterioration } \\
\text { of health, she has received repeated transfusion of convalescent plasma. After two cycles of convalescent } \\
\text { plasma intervention, the density of consolidation was gradually reduced and turned into scattered GGOs with } \\
\text { subpleural line. }\end{array}$ \\
\hline Case 3 & $\begin{array}{l}\text { A } 56 \text { years old man admitted in hospital positive COVID-19 patient with extensive lung lesions. Chest CT on } \\
\text { February } 21^{\text {st }} \text { showed consolidation in the right upper lobe and multiple GGOs in bilateral lungs. Reticular } \\
\text { opacities with vacuole inside and fibrosis streak were evident in the right down lobe. After three cycles of } \\
\text { convalescent plasma interventionas expected, serum IgM and IgG titers increased and complete resolution of } \\
\text { consolidation and GGO'S. }\end{array}$ \\
\hline Case 4 & $\begin{array}{c}\text { A 63-year-old women COVID-19 patient with Sjögren syndrome for } 10 \text { years. She was given Levofloxacin, } \\
\text { arbidol and oxygen treatment and repeated transfusion of convalescent plasma. Chest CT on March } \\
\text { 5th showed the resolution of solidified lesions, and the residual lesions presented as GGOs with partial } \\
\text { consolidation. }\end{array}$ \\
\hline
\end{tabular}

\section{Result}

The results of the selection and reviewing the Articles for this research analysis publication shows marked positive results. The results show the Traditional Chinese Medicine with western medicine or alone provide robust immunity to patients to fight against COVID-19. The measurement and statistical analysis by SPSS 19 diagrammatic presentation shows that majority of the patients get successful treatment by Traditional Chinese Medicine with western medicine or alone. A total of 93 effective Chinese medicine prescriptions and a total of 157 kinds of constituent medicines were collected. Data analysis was performed by SPSS 18.0 software [9]. The results showed that most of the medicines are with cold and warm properties, 69 with cold medicines, accounting for $43.95 \%$, 57 with warm medicines, accounting for $36.31 \%$, and 
less with hot medicines, taking up $1.27 \%$. The pungent medicines are mostly found in the early stage, the bitter drugs are the main flavor in the middle and severe stage, and the sweet medicines are mostly used in the recovery stage. The meridian of the drug is more concentrated at the lung, stomach, and heart, and most of drugs are into the lung meridian, accounting for $24.55 \%$ these medicines are mostly into the lungs and stomach in the initial and middle stages, and into the heart and kidney in severe stages. Most of the drugs have five types of functions treating exterior syndromes, resolving dampness, clearing heat, replenishing deficiency, resolving phlegm, cough, and asthma. In the early stage, both drugs for treating exterior syndromes and heat clearing drugs were equally used, accounting for $18.81 \%$ each [9].

The current 2019-nCoV outbreak is moving rapidly the cumulative number of confirmed cases in mainland China has reached 80151, with 47,204 (58.89\%) cured cases and 2943 (3.67\%) deaths as of 2-Mar-2020 and no specific drug has been discovered for Coronavirus Disease 2019 (COVID-19)(Ren JL, Zhang AH, Wang XJ, 2020). However, a number of clinical practice results showed that traditional Chinese medicine (TCM) plays significant role in the treatment of COVID-19 bringing new hope for the prevention and control of COVID-19.TCM has a long history and played an indispensable role in the prevention and treatment of several epidemic diseases. During the SARS epidemic in 2003 the intervention of TCM has also achieved remarkable therapeutic effect. During the treatment period of COVID-19 more than 3100 medical staff of TCM were dispatched to Hubei province and TCM scheme was included in the guideline on diagnosis and treatment of COVID-19 and TCM experts fully participate in the whole rescue process. From current treatment results TCM based on an over-all symptoms of 2019-nCoV pneumonia patients, has suggested to prescribe prescription that are likely to be effective(Ren JL, Zhang AH, Wang XJ, 2020).

\section{Discussion}

Novel coronavirus pneumonia, named as COVID-19 by the World Health Organization has spread widely since December 2019 with more than 40,000 confirmed cases in China and exportations to over 20 countries(Ling CQ, 2020).On January 30 2020, the World Health Organization declared the epidemic to be a public health emergency of international concern in the second meeting of the Emergency Committee. It was recommended that potential vaccines and antiviral medicines should be developed. However, the development of these therapeutics will take months, even years. For this specific indication, rapid performance of traditional Chinese medicine (TCM) can contribute as an alternative measure. It is a pivotal moment in defeating the current outbreak of the novel coronavirus. With the data published by Zhang's group, TCM practitioners around the world may speed up the experimental research and clinical use of these remedies, especially in those countries, territories or areas with reported and confirmed cases of COVID-19 [10].
In order to evaluate the efficacy of convalescent plasma therapy in COVID-19 patients we did this timely descriptive study [11]. 6 laboratory confirmed COVID-19 patients were enrolled and received the transfusion of $\mathrm{ABO}$ compatible convalescent plasma. Transfusion of convalescent plasma led to a resolution of ground glass opacities (GGOs) and consolidation in patient \#1, \#2, \#3, \#4 and \#6. In patient \#1 and \#5 who presented with SARS-CoV-2 in throat swab, convalescent plasma therapy elicited an elimination of virus. Serologic analysis indicated an immediate increase in anti-SARS-CoV-2 antibody titers in patient \#2 and \#3, but not in patient \#1. This study indicates that convalescent plasma therapy is effective and specific for COVID-19. This intervention has a special significance for eliminating SARS-CoV-2 and is believed to be a promising state-of-art therapy during COVID-19 pandemic crisis [7].

The use of Chinese Medicine (CM) to prevent epidemics of infectious diseases was traced back to ancient Chinese practice cited in Huangdi's Internal Classic (Huang Di Nei Jing) where preventive effects were recorded [12]. There were 3 studies using $\mathrm{CM}$ for prevention of SARS and 4 studies for H1N1 influenza. None of the participants who took CM contracted SARS in the 3 studies. The infection rate of H1N1 influenza in the CM group was significantly lower than the non-CM group (relative risk $0.36,95 \%$ confidence interval 0.24-0.52: n=4). For prevention of COVID-19, 23 provinces in China issued CM programs. The main principles of $\mathrm{CM}$ use were to tonify qi to protect from external pathogens, disperse wind and discharge heat, and resolve dampness. Based on historical records and human evidence of SARS and H1N1 influenza prevention Chinese herbal formula could be an alternative approach for prevention of COVID-19 in high-risk population. Prospective, rigorous population studies are warranted to confirm the potential preventive effect of CM [12].

Pneumonia associated with the 2019 novel coronavirus (2019$\mathrm{nCoV}$ ) is continuously and rapidly circulating at present [12]. No effective antiviral treatment has been verified thus far. We report here the clinical characteristics and therapeutic procedure for four patients with mild or severe 2019-nCoV pneumonia admitted to Shanghai Public Health Clinical Center. All the patients were given antiviral treatment including lopinavir/ritonavir (Kaletra), arbidol, and Shufeng Jiedu Capsule (SFJDC, a traditional Chinese medicine) and other necessary support care. After treatment three patients gained significant improvement in pneumonia associated symptoms two of whom were confirmed 2019-nCoV negative and discharged and one of whom was virus negative at the first test. The remaining patient with severe pneumonia had shown signs of improvement by the cutoff date for data collection. Results obtained in the current study may provide clues for treatment of 2019-nCoV pneumonia. The efficacy of antiviral treatment including lopinavir/ ritonavir, arbidol, and SFJDC warrants further verification in future study [13]. 


\section{Conclusion}

The main theme of this Article is to discuss the Preventions, training and control strategies against COVID-19 novel corona virus in China in initial first two months of Pandemic. When Chinese health authorities in Wuhan china found Novel coronavirus pneumonia (NCP) in patients in health facilities they first treated them with (TCM) Traditional Chinese medicines with western medicines. In most of the cases they have found (TCM) Traditional Chinese medicines more effective than other medicines. The current positive statistics of Pandemics about China shows the complete control on COVID-19 with no spread and no Fatalities in May 2019. While the Pandemic of COVID-19 is on the rise all around the world specially in the USA, Europe and the UK [14-16].

\section{Conflict of Interest}

The author declares that there is no conflict of interest statement.

\section{References}

1. Zhonghua Liu, Xing Bing, Xue Za Zhi. (2020) An update on the epidemiological characteristics of novel coronavirus pneumonia (COVID-19). The Chinese Preventive Medicine Association Beijing 100021, China 41(2): 139-144.

2. Hong-Zhi DU, Xiao-Ying HOU, Yu-Huan MIAO, Liu DH (2020) Traditional Chinese Medicine: an effective treatment for2019 novel coronavirus pneumonia (NCP). Chinese Journal of Natural Medicines 18(3): 206-210.

3. National library of medicine (2020) Research progress of intervention of Chinese herbal medicine and its active components on human coronavirus.

4. Liang H, Acharya G (2020) Novel corona virus disease (COVID-19) in pregnancy: What clinical recommendations to follow. Obstetrics and Gynaecology special editorial 99(4): 439-442.

ISSN: 2574-1241

DOI: 10.26717/BJSTR.2020.27.004544

Ahsan Ali Siddiqui. Biomed J Sci \& Tech Res

(C) This work is licensed under Creative

Submission Link: https://biomedres.us/submit-manuscript.php
5. Sun L, Shen L, Fan J, Gu F, Hu Y, et al. (2020) Clinical Features of Patients with Coronavirus Disease 2019 (COVID-19) from a Designated Hospital in Beijing, China 395(10223): 497-506.

6. Jin YH, Cai L, Cheng ZS, Hong Cheng, Tong Deng, et al. (2020) A rapid advice guideline for the diagnosis and treatment of 2019 novel coronavirus (2019-nCoV) infected pneumonia (standard version). BMC Military Medical Research 7(1): 4.

7. Wikipedia Article (2020) Literature review, for a focused scientific review with pre-defined methodology see Systematic review.

8. Luo E, Zhang D, Luo H, Bowen Liu, Keming Zhao, et al. (2020) Treatment efficacy analysis of traditional Chinese medicine for novel coronavirus pneumonia (COVID-19): an empirical study from Wuhan, Hubei Province, China. Chin Med 15: 34.

9. Gu M, Liu J, Shi NN, Li XD, Huang ZD, Wu JK, et al. (2020) Analysis of the efficacy of traditional Chinese medicine in preventing and treating newtype coronavirus pneumonia. CnKi.net Journal 45(6): 1253-1258.

10. Ling CQ (2020) Traditional Chinese medicine is a resource for drug discovery against 2019 novel coronavirus (SARS-CoV-2). Journal of Integrative Medicine 18(2): 87-88.

11.Wiley Library Journal of Medical Virology (2020) Treatment with convalescent plasma for COVID-19 patients in Wuhan, China.

12. Hui Luo, Qiao-ling Tang, Ya-xi Shang, Liang SB, Yang M, et al. (2020) Can Chinese Medicine Be Used for Prevention of Corona Virus Disease 2019 (COVID-19)? A Review of Historical Classics, Research Evidence and Current Prevention Programs. Springerlink Chinese Journal of Integrative Medicine 26(4): 243-250.

13. Wang Z, Chen X, Lu Y, Yan W, Yang D, et al. (2020) Clinical characteristics and therapeutic procedure for four cases with 2019 novel coronavirus pneumonia receiving combined Chinese and Western medicine treatment. J Stage $20^{\text {th }}$ Bioscience trends 14(1): 64-68.

14. Ren JL, Zhang AH, Wang XJ (2020) Traditional Chinese medicine for COVID-19 treatment. ELSEVIER Pharmacological research 155: 104743.

15. Hongzhou Lu (2020) Drug treatment options for the 2019-new coronavirus (2019-nCoV). J Stage 20th Bioscience trends 14(1): 69-71

16. IBM (2006) IBM SPSS Software.

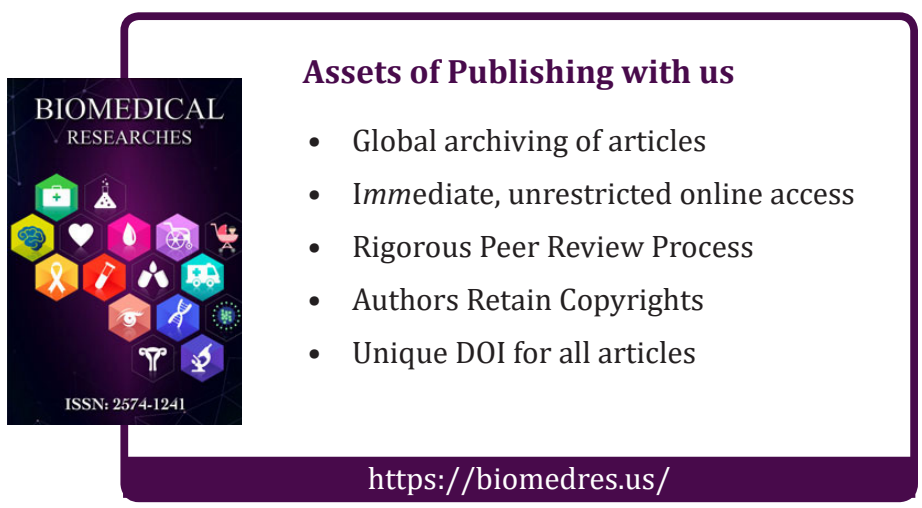

